\title{
La diversidad en las organizaciones indigenas del Cauca: El Consejo Territorial de Autoridades Indigenas del Oriente Caucano ${ }^{1}$
}

\author{
Santiago Andrés Gutiérrez Sánchez ${ }^{2}$ \\ Investigador Independiente \\ gutierrez.santiago.andres@gmail.com \\ Recibido: 13 de febrero de 2014 \\ Aceptado: 7 de julio de 2014 \\ Disponible en línea: 15 de marzo de 2015
}

\footnotetext{
${ }^{1}$ Artículo de reflexión. Este artículo presenta una reflexión y un aporte teórico a las discusiones contemporáneas sobre la identidad étnica y la etnicidad en las organizaciones indígenas de Colombia. Para esto se retoman los resultados de campo y de investigación elaborados por el autor en su tesis de pregrado, pero se presentan desde un planteamiento conceptual diferente. $\mathrm{El}$ artículo no fue financiado por ninguna institución.

2 Antropólogo de la Pontificia Universidad Javeriana
} 


\title{
La diversidad en las organizaciones indigenas del Cauca: E1 Consejo Territorial de Autoridades Indigenas del Oriente Caucano
}

\section{Resumen}

La intención de este artículo es exponer, desde un caso específico, la multiplicidad de propósitos y discusiones que hay entre las organizaciones indígenas del Cauca, Colombia. Retomo el concepto de etnicidad para entender a las organizaciones indígenas como escenarios en los que se reconstruyen los procesos étnicos, se redefinen relaciones y se pone en discusión la manera de reconocer la diversidad. No obstante, este texto se concentra exclusivamente en las diferentes expresiones y relaciones que se dan dentro de las organizaciones indígenas, especialmente aquellas germinadas después de la constitución del 1991. Para ello expongo la manera en que surge el Programa de Educación del Consejo Territorial de Autoridades Indigenas del Oriente Caucano (COTAINDOC) y sus principales propuestas. Concluyo que este programa redefine su manera de reconocer la diversidad cultural y las relaciones con otras organizaciones e instituciones.

Palabras clave: diversidad cultural; etnicidad; organizaciones indígenas; educación indígena

\section{Diversity in the Indigenous Organizations from Cauca: The Eastern Cauca Territorial Council of Indigenous Authorities}

\begin{abstract}
The intent of this article is to present, from a specific case, the multiplicity of intentions and discussions between indigenous organizations from Cauca, Colombia. I go back to the concept of ethnicity in order to understand indigenous organizations as arenas where ethnic processes are reconstructed, relationships are redefined and the way diversity is recognized is questioned. However, this paper focuses exclusively on the different expressions and relationships that exist within indigenous organizations, especially those created after the Constitution of 1991. For this purpose I present how the Education Program of the Eastern Cauca Territorial Council of Indigenous Authorities (COTAINDOC) and their main proposals emerged.I conclude that this program redefines its way of recognizing cultural diversity and relationships with other organizations and institutions.
\end{abstract}

Keywords: cultural diversity; ethnicity; indigenous organizations; Indigenous education

\section{Diversidade nas organizações indigenas do Cauca: Conselho Territorial de Autoridades Indigenas do Este Caucano}

\section{Resumo}

A intenção deste artigo é expor, desde um caso específico, a multiplicidade de propósitos e discussões que existem entre as organizações indígenas do Cauca, Colômbia. Retomo o conceito de etnicidade para entender as organizações indígenas como cenários em que os processos étnicos são reconstruídos, redefinem-se relações e põe-se em discussão a maneira de reconhecer a diversidade. Contudo, este texto centra-se exclusivamente nas diferentes expressões e relacionamentos que se dão dentro das organizações indígenas, especialmente aquelas germinadas depois da constituição de 1991. Para isso exprimo a maneira em que surge o Programa de Ensino do Conselho Territorial de Autoridades Indígenas do Oriente Caucano (COTAINDOC) e suas principais propostas. Concluo que esse programa redefine a maneira de reconhecer a diversidade cultural e as relações com outras organizações e instituições.

Palavras-chave: diversidade cultural; etnicidade; organizações indígenas; ensino indígena 


\section{Introducción}

Aunque el espacio territorial definido como Colombia ha encerrado la presencia de diferentes grupos y sociedades humanas desde hace más de 200 años, los cuales han establecido algún tipo de relaciones con la sociedad mayoritaria, es a partir de 1991 que el Estado nacional reconoce a esta como un territorio multicultural y pluriétnico. Durante años, la relación entre los pueblos indígenas y la sociedad nacional era explícitamente de exclusión, marginalización y aculturación, sin embargo la Constitución de 1991 reconoció varios derechos sociales y culturales de los grupos étnicos, como el derecho a la existencia, al territorio, al ejercicio de la diferencia y al desarrollo de una autonomía. Lo anterior no quiere decir que las relaciones entre los pueblos indígenas y la sociedad sean equitativas, pero sí plantea la necesidad de que tanto las instituciones públicas y privadas como la sociedad en su conjunto reconozcan e interactúen con la diversidad cultural. Con esta intención las organizaciones indígenas se vuelven centrales, pues aunque la carta constitucional tiene incidencia en las luchas sociales de la década de 1960 y 1970, es en el periodo post-constitucional que estas organizaciones se convierten en actores elementales para el desarrollo de la politica cultural del Estado nacional y la articulación entre las instituciones públicas y privadas y los pueblos indígenas.

No obstante, la diversidad cultural se refiere a la multiplicidad de formas en que se expresan las culturas de los pueblos y sociedades, entramando dinámicas de tensiones, presiones mutuas, fricciones y a veces, valores y estilos de vida (Díaz-Polanco, 2009, p. 19). Un ejemplo de lo anterior es la forma de referirse a los pueblos indígenas, en la que existe una diferencia entre comunidad o grupo étnico minoritario y la noción de pueblo o nacionalidad, pues la primera integra diferentes localidades y personas mientras el vocablo pueblo establece una noción más amplia en donde conviven varias comunidades con complejos sistemas de organización (Díaz-Polanco, 2009). Justamente, las diferentes organizaciones indígenas en Latinoamérica han señalado esta distinción y han encaminado su trasegar en reconstituirse como Pueblos y no solamente como grupos étnicos minoritarios (Díaz-Polanco, 2009, pp. 24-25); toda vez que la manera de entender 
la diversidad y el reconocimiento étnico influyen tanto en la manera en que se identifican a sí mismos los grupos y/o individuos, como en el tipo de tratamiento que tiene la autonomía o la educación -entre otros aspectos- en las organizaciones, las comunidades indígenas y la sociedad mayoritaria.

Tomando en consideración lo anterior, las organizaciones indígenas han entrado en los discursos y dinámicas de la diversidad cultural, pues al estar esta permeada por relaciones de poder e intereses particulares de diferentes actores, intervendrá en el desarrollo de los procesos organizativos indígenas y sus reivindicaciones. Por ello es necesario entender cómo opera la diversidad dentro de las mismas y por consiguiente, este artículo pretende ser un insumo a la comprensión de la manera como es tratada la diversidad cultural dentro los procesos organizativos indígenas, especialmente aquellas germinadas después de la constitución de 1991. Para esto se analizan algunos aspectos importantes del surgimiento y desarrollo del Programa de Educación del Consejo Territorial de Autoridades Indígenas del Oriente Caucano (COTAINDOC) como un caso en donde se puede mirar la implicación que tiene el tratamiento de la diversidad y el reconocimiento étnico dentro de los procesos organizativos indígenas.

El COTAINDOC es una organización zonal interétnica del oriente del Cauca, entre los municipios de Totoró, Piendamó, Silvia y Morales, la cual reúne a cinco pueblos indígenas (Nasas, Misak, Ambalueños, Polindaras y Kizgueños o Kichuz) alrededor de once comunidades (Raíces de Oriente, San Antonio y La María, Quichaya, Jebalá, La Gaitana, Tumburao, Kizgó, Pitayó y Ambaló, Polindara y Paniquitá).

El presente artículo está expuesto de la siguiente manera: primero se hace un recuento histórico de las organizaciones indígenas del Cauca y una breve exposición del tratamiento que ha tenido el concepto de etnicidad en la antropologia; luego se expone el surgimiento y desarrollo del Programa de Educación del COTAINDOC mediante el análisis de dos de sus principales proyectos: el trabajo con maestros(as) y el PEC; y finalmente se presentan algunas consideraciones frente al caso específico del COTAINDOC. 


\section{La etnicidad, las organizaciones indigenas y la diversidad}

Frente a palabras como tribu o raza, que usualmente eran empleadas para referirse a los grupos humanos diferentes de las sociedades occidentales, surgiría el término de grupos étnicos, el cual se empezó a usar después de la Segunda Guerra Mundial (Barfield, 2001/1997, p. 328). Si bien los primeros trabajos que utilizarian este concepto desde la antropología intentaban alejarse de perspectivas biológicas y evolucionistas, estos seguían haciendo referencia a cierto tipo de poblaciones no occidentales o minoritarias que no tenían aparentemente, ninguna relación con un Estado, ni con una sociedad nacional mayoritaria y los cuales, a partir de este término, sujetaban todas las prácticas y organizaciones sociales y culturales de una sociedad. No obstante, el concepto de grupos étnicos tuvo un desarrollo distinto en el trabajo de Fredrik Barth (1976/1969). Barth plantearía que lo étnico se construye mediante la organización de las relaciones interétnicas entre diferentes grupos, mas no es el resultado de la correspondencia de ciertos aspectos socioculturales definidos y un grupo específico (Barth, 1976/1969). Es decir, que la manera como se construye lo étnico y la manera como los grupos étnicos se ven a sí mismos se da a partir de la forma en que se organicen las fronteras étnicas y las relaciones entre diferentes grupos humanos.

Un espacio en donde se encuentran los diferentes grupos humanos y en donde se (re)configuran las fronteras étnicas es la escuela. Las escuelas que se encontraban en territorios indigenas estuvieron en manos de las instituciones eclesiásticas, las cuales eran responsables de la educación que se le impartía a las poblaciones indigenas y afros (CRIC, 2011, p. 23). Alli las lenguas y expresiones indigenas eran vistas como aspectos negativos e incivilizados que se consideraba necesario eliminar a partir de la educación (Gros y Morales, 2009). Así las escuelas eran espacios en donde las poblaciones indígenas se veían introducidas en dinámicas de exclusión, trasformación, extinción y dominación (Pancho, 2007).

En 1971 surge el Consejo Regional Indígena del Cauca (CRIC) -primera organización indígena regional en el departamento- que plantaría 
en principio, siete puntos de lucha dentro de los cuales estaban la recuperación de tierras, la constitución de cabildos, el fortalecimiento de las lenguas indígenas y la formación de maestros indígenas. Además de esto, en 1978 esta organización crearía el Programa de Educación Bilingüe e Intercultural (PEBI) con el objetivo de desarrollar una educación que promoviera la recuperación de la identidad, el territorio y las prácticas culturales (CRIC, 2004). Por tanto, las primeras organizaciones indígenas del $\mathrm{Cauca}^{3}$ consideraban necesario cambiar el modelo educativo y la representación o trato que allí se daban sobre la población indígena ${ }^{4}$.

Estas organizaciones indígenas intentaban modificar, en términos de Barth (1976/1969), el tipo de relaciones e intercambios que se habian establecido entre los pueblos indígenas y la sociedad mayoritaria, sin embargo una critica al planteamiento de Barth (1976/1969) fue su escaso acercamiento histórico y socioeconómico al momento de analizar las fronteras étnicas. No obstante algunas investigaciones sobre los pueblos indígenas caucanos manifestarian una idea similar, afirmando que aunque eran los componentes del Estado los que determinaban lo que se considera como indigena, era necesario aceptar una presencia y una lucha de los 'grupos minoritarios' dentro de la nación moderna para definir qué es lo indígena (Friede, 1972, p. 152).

Si bien antes del surgimiento de estas organizaciones se habian establecido relaciones y sistemas de representación entre los pueblos indígenas y la sociedad nacional, es en las décadas de 1960 y 1970 que se puede ubicar el surgimiento de un sujeto colectivo que revindicará su afirmación étnica ante el Estado, intentando trasformar las

3 Durante la década de 1970 las comunidades nasa, misak o guambianos, kokonucos y yanaconas confluian en el Consejo Regional Indigena del Cauca, sin embargo a principios de la década de 1980, debido a discusiones y disputas internas, se crearía el Movimiento de Autoridades Indigenas (AICO) que en principio reunía a algunos cabildos y resguardos de los pueblos misak (Consultar más al respecto en Vasco, 2002; Caviedes, 2002; Laurent, 2005).

4 En la década de 1980 surgirian algunas iniciativas comunitarias de educación propia o indígena en los territorios en donde se habian gestado recuperaciones de tierra. Estas iniciativas eran auto-gestionadas por las comunidades y apoyadas por las organizaciones indígenas regionales (CRIC o AICO). Las escuelas comunitarias nasas se dieron en López Adentro, Laguna de Siberia, Las Delicias y el Cabuyo, entre otras y en el resguardo de Guambia en el Chiman-La Marquesa (CRIC, 2004, pp. 40-41). En el resguardo de Ambaló, municipio de Silvia, también se creó una escuela comunitaria en la vereda de Agoyán. 
relaciones que tenía la sociedad nacional históricamente con respecto a los pueblos indígenas (Gros, 1991; Houghton y Villa, 2005). No obstante, aunque las reivindicaciones y experiencias del CRIC alrededor de la educación -entre otros aspectos- expresen la articulación que hubo entre los procesos organizativos indígenas y las luchas por un reconocimiento étnico, no se puede afirmar que estas organizaciones representaban y buscaban una identidad étnica uniforme. Desconoceríamos las diferentes discusiones y disputas que se han desarrollado dentro de las organizaciones y comunidades indígenas del Cauca $^{5}$. Entonces sería erróneo desarrollar una mirada homogénea y estática sobre las organizaciones indígenas, sin comprender las implicaciones del surgimiento de este sujeto colectivo y del reconocimiento de lo étnico por parte del Estado.

Las organizaciones indigenas en Colombia han atravesado diferentes momentos de relacionamiento con el Estado y con la sociedad nacional, lo que en cierta medida ha generado el surgimiento de un abanico de organizaciones indígenas. El primer momento se dio con el surgimiento de las organizaciones indígenas regionales ${ }^{6}$ y culminaría con el surgimiento de las organizaciones a nivel nacional ${ }^{7}$. Pero lo vivido durante las décadas de 1970 y 1980 se trasformaría con la Constitución de 1991, momento en que las organizaciones indígenas y la diversidad cultural fueron reconocidas por el Estado. A partir de este periodo las organizaciones indígenas vivieron una época de optimismo debido a los derechos constitucionales ganados y experimentaron nuevos espacios de discusión y nuevas formas de relación con el Estado (Houghton y Villa, 2005).

5 Durante sus primeros diez años, el CRIC reunió a la mayoría de los pueblos indígenas del Cauca, participando en mayor cantidad, cabildos y comunidades de los pueblos nasa, kokonucos, yanacona y misak. Sin embargo a principios de la década de 1980 surgió otra organización indígena de carácter regional y nacional denominada Movimiento de Autoridades Indígenas, que posteriormente cambió su nombre al de Autoridades Indigenas de Colombia (AICO). Esta nueva organización surgió tras una serie de discusiones y confrontaciones entre algunas comunidades indígenas del Cauca (como Guambia del pueblo misak y Jambaló y Pitayó del pueblo nasa) y la estructura organizativa del CRIC (Caviedes, 2002; Laurent, 2005).

6 Como el CRIC, la Organización Regional Embera Wauunan, la Organización Indígena de Antioquia, entre otras.

7 Las organizaciones indígenas de carácter nacional, como la Organización Indígena de Colombia y las Autoridades Indigenas de Colombia, surgirian y se fortalecerian durante la década de 1980 y 1990. 
Algunas de estas trasformaciones fueron la transferencia de recursos económicos a las comunidades indígenas; la posibilidad de que los cabildos indígenas se asocien y puedan ejecutar recursos y/o proyectos estatales (Decreto 1088 de 1993); la obligación por parte del Estado de realizar cualquier proyecto en territorio indígena acorde al entorno político, social y cultural de los grupos étnicos; y la necesidad de que los pueblos indígenas participen en la construcción de los proyectos educativos de las instituciones educativas que se encuentran en sus territorios. Así las cosas, en el departamento del Cauca surgieron varias organizaciones zonales como la Asociación de Cabildos Indígenas del Norte del Cauca, la Asociación de Cabildos de Juan Tama y el Consejo Territorial de Autoridades Indígenas del Oriente Cauca -entre otras, con el propósito de desarrollar proyectos sociales y culturales encaminados a fortalecer la autonomía, la cultura y la unidad de los pueblos indígenas.

Al momento de surgir, algunas de estas organizaciones tenían el propósito de conformar paulatinamente, una entidad territorial indige$n a$, pues allí se reunían varias comunidades indígenas con las cuales se trabajaba la elaboración de los planes de vida y los proyectos educativos comunitarios (PEC) (Rueda, Gomez y Martinez, 2003).

Este reconocimiento étnico y el abanico de nuevas relaciones/ espacios influyeron tanto en los procesos organizativos y de identificación que vivian los pueblos indígenas, como en sus reivindicaciones, al tiempo que las organizaciones indígenas se involucraron en las dinámicas que les eran impuestas desde la institucionalidad estatal (como la constante concertación y la formulación de políticas públicas) y la lógica del gobierno indirecto ${ }^{8}$. Así, se puede ver como cada vez más, ciertos, espacios organizativos indígenas destinados a la elaboración de la política pública, la prestación de los servicios públicos y/o el control territorial; como por ejemplo, los nuevos trabajos de gestión y gasto de recursos públicos que tenían y tienen que hacer los cabildos indigenas.

8 La lógica de gobierno indirecto es la manera como el Estado despliega su quehacer de manera indirecta a partir de actores o sujetos intermedios. En este caso el Estado pretende ahondar en la legitimidad estatal y desarrollar su control territorial a partir de las organizaciones indigenas (Gros, 1997). 
De igual manera la tensión generada sobre qué es lo indígena se trasladaría a los procesos de construcción y reconocimiento de las nuevas organizaciones indigenas, ya que la definición y el quehacer que la normatividad desarrollaria sobre estas nuevas organizaciones indígenas post-constituyentes no tendrian en cuenta los propósitos de las organizaciones y pueblos indígenas. Por un lado, el Estado define a este nuevo tipo de organizaciones como asociaciones de cabildos indígenas, las cuales simplemente le permiten a las comunidades indígenas agruparse y ejecutar recursos públicos o privados (Decreto 1088); pero de otro lado, el CRIC afirmaría que el propósito de estas nuevas organizaciones era fortalecer a las comunidades indígenas, mejorar la articulación entre estas y el movimiento indígena regional, enfrentar las nuevas dinámicas que viven las comunidades indígenas a raíz del reconocimiento étnico y constituirse, en un futuro, como entidades territoriales indígenas (CRIC, 2006).

Algunos estudios pondrán a la luz de este nuevo contexto el hecho de que la identidad y la etnicidad son un instrumento o recurso político y social que algún grupo o movimiento social desarrolla en una estructura social más amplia (Hall, 2003; Neira, 2008). Un trabajo desde esta perspectiva lo hace Christian Gros (1997), quien afirmará que las organizaciones indígenas tienen dos posiciones en el marco del reconocimiento étnico. Por un lado, las organizaciones indígenas hacen uso instrumental de la identidad y/o diferencia con el objetivo de obtener el reconocimiento de derechos particulares y la defensa de intereses colectivos, lo que hace de estas un escenario para la integración y la modernización sin pasar por la asimilación o mestizaje (Gros. 1997).

No obstante por otro lado, al estar los procesos de reconocimiento étnico de los pueblos indigenas envueltos en aspectos (leyes, políticas públicas, ONG, etc.) que lo vinculan a las dinámicas de las relaciones e instituciones estatales y no gubernamentales, las organizaciones indígenas se convierten entonces en ese nuevo actor étnico que responde a la legitimidad estatal y al gobierno indirecto (Gros, 1997). Esto lleva a que las organizaciones indígenas sean indispensables para el desarrollo de la nueva política del Estado, ya que allí se despliegan los espacios en los que los derechos culturales y sociales serán tratados, 
como las mesas de concertación, los proyectos inter-institucionales y los programas de etnoeducación o salud (Gros, 1999).

Si bien esta perspectiva es interesante, su análisis solo explica un fenómeno mediante la intención estratégica de un sujeto dentro de una estructura de oportunidad, sin comprender a profundidad los sistemas de valores y significados que hay dentro de las diferentes organizaciones indígenas.

Lo anterior no significa que vea las organizaciones indigenas por fuera de las relaciones con el Estado y la sociedad mayoritaria; de hecho considero importante analizar la relación que hay entre estas y las políticas estatales. Pero no se puede suponer que hay relaciones uniformes entre las mismas organizaciones indigenas y, mucho menos, entre las diferentes organizaciones indígenas (cabildo, asociación, organización regional y nacional) y las instituciones estatales. Por ello, la intención de este artículo es plantear que no es pertinente partir de una mirada que homogenice a las organizaciones indígenas, sus espacios y sus proyectos.

Al contrario, es importante entender que la cuestión étnica y los proyectos y reivindicaciones de los movimientos indígenas del Cauca tienen un carácter múltiple e intercultural, ya que las organizaciones son una compleja rama de redes interétnicas que no parten de especializar su identidad étnica, sino del desarrollo de múltiples identidades y de una diversidad de planteamientos políticos inter-conectados (Rappaport, 2008). Considero entonces necesario plantear el análisis en términos de una multiplicidad de espacios, propósitos y relaciones dentro de las mismas organizaciones indígenas. Esta variedad se moldea y se establece a partir de los procesos de construcción y reconocimiento que viven las mismas organizaciones indígenas, más allá de la relación CRIC-Estado.

Para lograr lo anterior es necesario desarrollar un análisis que le dé prioridad a los cambios contextuales, a las relaciones y a los espacios que tienen lugar en las organizaciones indígenas. Para esto retomo el planteamiento del antropólogo Bonfil Batalla, que asevera que la relación entre la etnicidad y las organizaciones indígenas no es algo pasivo ni estático, sino que al contrario, es algo que vive en tensión 
constantemente, creando y recreando la cultura, reforzando los ámbitos propios e integrando aspectos ajenos que permitan expresar y renovar la identidad étnica (Bonfil, 1987, p. 11).

Un análisis desde esta perspectiva lo expone Maria Teresa Findji (1993) que encuentra que el fortalecimiento de las comunidades y de los cabildos no son sinónimo de un mismo proceso, al percibir que después de la etapa de recuperación de tierras en los años setenta, varios cabildos expusieron sus debilidades y tensiones. Algunos cabildos acudieron a entidades públicas y organismos internacionales para conseguir apoyo económico y empezar a generar proyectos sociales y culturales, lo que generó nuevas dinámicas externas a los cabildos, como el trabajo de repensar los nuevos problemas (trasferencias, trámites y reuniones con instituciones externas) desde adentro de la comunidad y produciendo inconvenientes en la relación cabildo-comunidades (Findji, 1993, p. 67).

Lo interesante del planteamiento de Findji (1993) es que manifiesta que aquellas tensiones constantes que hay en los procesos organizativos indígenas se configuran a partir de una lógica contextual. Ella plantea que la lógica de la recuperación de tierras que caracterizó al movimiento indígena del Cauca durante las décadas de 1970 y 1980 obedeció a una lógica de la defensa cultural para la supervivencia colectiva, pero que en el contexto actual (periodo post-constitucional), las organizaciones se enfrentan a una trasformación por suceder (hacer), pues ya no hay un contexto de resistencia del movimiento indígena sino una lógica de reconstrucción en tiempos de reconocimiento étnico (Findji, 1993, p. 64). Es decir que los referentes culturales, las prácticas y las reivindicaciones de las organizaciones indígenas se (re)construyen debido a la lógica contextual actual, la del reconocimiento étnico.

Estas tensiones y lógicas organizativas se materializan a partir del proceso de etnicidad que llevan (construyen) los pueblos indígenas. Para el antropólogo mexicano Miguel Bartolomé, la etnicidad constituye la expresión contextual y práctica de los procesos de identificación y de la construcción de una conciencia colectiva étnica (Bartolomé, 2004/1997, p. 64). Si bien la etnicidad no solo se manifiesta en los 
movimientos indígenas, este artículo se concentra en el contexto y las dinámicas que tienen lugar en los procesos organizativos indigenas.

Precisamente las diversas reivindicaciones y proyectos de las organizaciones indígenas configurarian lo que Bartolomé denomina construcción nacionalitaria, que sería la búsqueda por construir sujetos y espacios colectivos que apelan a una identidad y acción social compartida, basada en una tradición cultural propia o apropiada y que pretende relacionarse en términos igualitarios con los otros conjuntos culturales del Estado (Bartolomé, 2006, p. 223). En este caso la educación ha sido un escenario de disputas entre las organizaciones indígenas, la sociedad nacional y las instituciones estatales por la reproducción y el fortalecimiento de las expresiones culturales o del status quo; por el reconocimiento étnico; y por la construcción de una conciencia colectiva étnica o nacionalitaria. En términos de Bartolomé por lo tanto, la etnicidad aquí nos permitirá entender la manera en que se dan las expresiones contextuales y prácticas que tienen lugar en el Programa de Educación del COTAINDOC, permitiéndonos comprender el lugar que tiene la diversidad en este caso y la manera en que es tratada.

\section{La legitimidad organizativa y el Programa de Educación del COTAINDOC}

El Consejo Territorial de Autoridades Indígenas del Oriente Caucano (COTAINDOC) es una organización interétnica de carácter zonal -que surgió en 1999 apadrinada por el CRIC-, la cual reúne a las comunidades del pueblo nasa de Raíces de Oriente, Quichaya, Jebalá, La Gaitana, Tumburao, Pitayó y Paniquitá; las comunidades del pueblo misak de San Antonio y La María; y las comunidades de kizgó o Kichuz, de Ambaló y la comunidad de polindara (autodenominados polindaras). Dichas comunidades se encuentran ubicadas al suroccidente colombiano, en la cordillera central y en el llano interandino o, sabana ligeramente ondulada, en los municipios de Silvia, Totoró, Piendamó y Morales. 
El COTAINDOC surgiría como respuesta a los pocos espacios organizativos y de articulación que existían a finales del siglo XX entre las comunidades indígenas de la zona oriente, a excepción del Cabildo Mayor de Guambia. Alli después de las primeras recuperaciones de tierras, durante las décadas de 1970 y 1980, germinarian algunos espacios de trabajo alrededor de proyectos productivos y de la eliminación de cultivos ilícitos entre las comunidades de Ambaló, Polindara, Paniquita, Kisgó, Quichaya, Pitayó, Polindara, Jebalá, Tumburao y La María. Estas experiencias llevarian a los cabildos indígenas a conformar en 1993, la Junta Administradora de Proyectos, avanzando solamente en la ejecución de recursos para proyectos económicos hasta 1998. Sin embargo, en el caso del tema educativo no existían directrices ni propuestas conjuntas entre los cabildos indígenas, más allá de las adelantadas por el CRIC (entrevista a Alirio Sánchez, 2012).

En el primer congreso del COTAINDOC (2000), los principales problemas educativos que se identificaron entre las comunidades indígenas de la zona oriente fueron el desarrollo de los currículos y proyectos pedagógicos oficiales; la falta de capacitación de los padres de familia sobre el Proyecto Educativo Comunitario; y la ausencia de equipos de educación en la mayoría de cabildos y de espacios que permitieran intercambiar experiencia sobre la educación indígena (COTAINDOC, 2000a). Para enfrentar las anteriores situaciones se propone crear el programa de educación zonal del COTAINDOC; cuyo primer coordinador fue Julio Almendra, su labor era "identificar las políticas propias para hacer un proceso de sensibilización de la educación propia por el periodo de dos años" (Programa de Educación, 2010).

Aunque tenían la intención de empezar realizando un proceso de sensibilización, Julio Almendra desarrolló un diagnóstico en el cual se observaba que la mayoría de los procesos educativos que se daban en los territorios indígenas respondian al sistema educativo estatal y no a los procesos comunitarios de las comunidades (COTAINDOC, 2002). No obstante Eliodoro Quijano, coordinador del programa para el 2004, recuerda que su trabajo se concentraria en las relaciones con el CRIC y en la consolidación de una información real de las instituciones educativas, haciendo del trabajo del programa algo más estadístico y comunicativo (entrevista a Eliodoro Quijano, 2012). Así el objetivo del 
diagnóstico había cambiado al concentrarse en la elaboración de una estadística de los niños y niñas indígenas que eran atendidos en los establecimientos educativos.

Es necesario reconocer, antes de analizar cada propuesta, que la organización indígena regional fue un sujeto clave en los espacios que empezó a abrir el COTAINDOC. Durante 1997 y 2005 el CRIC le exigió al Estado reconocer y operativizar el Sistema Educativo Indígena Propio (SEIP), movilizaciones que dieron como resultado una serie de convenios de contratación docente para territorios indígenas entre el Programa de Educación Bilingüe e Intercultural (PEBI) del CRIC, la Secretaria de Educación Departamental y el Ministerio de Educación Nacional (CRIC, 2011). Estos convenios se hacian a partir de las estadísticas de cuántos niños, niñas y docentes habían en cada institución educativa, trabajo que realizaba el Programa de Educación para la zona oriente del Cauca (entrevista a Eliodoro Quijano, 2012). Entonces el Programa de Educación surgió acompañando el trabajo que venía haciendo el PEBI en los resguardos de la zona oriente. Esto implicó que las políticas educativas y los espacios agenciados por el programa fueran permeados por la organización indígena regional.

\section{Los(as) docentes y maestros(as)}

Si bien los(as) docentes eran un punto de referencia para las comunidades indígenas del Cauca antes de la aparición del COTAINDOC ${ }^{9}$, el trabajo adelantado por el PEBI y por el Programa de Educación generó que los profesores, los rectores y directores de núcleo ${ }^{10}$ aparecieran dentro de los discursos y discusiones del COTAINDOC como ejes problemáticos de la educación e identidad indígena. Es decir, se percibía que los docentes dificultaban el ejercicio de los derechos sociales y culturales reconocidos para los pueblos indígenas. Esta identificación no fue producto de una representación concentrada en la narración mitológica del territorio o en la cosmología indígena, sino que fue el

\footnotetext{
Por ejemplo, dentro de los primeros siete puntos de lucha del CRIC -en su plataforma de políticase enuncia la necesidad de formar docentes indigenas.

${ }^{10}$ Coordinador de varias instituciones educativas en un Municipio.
} 
resultado de un análisis sobre las relaciones históricas entre la educación y las comunidades indígenas (los diagnósticos).

Pero la relación con los(as) docentes se agudizaría con la reorganización de la educación que hizo la Ley 715 de 2001, la cual cambió la lógica de la educación centralizando las funciones y proyectos educativos en los directores de núcleo y en la SED (Entrevista a Eliodoro Quijano, 2012). Esto llevó al COTAINDOC a reafirmar que los docentes y rectores no se articulaban a las orientaciones de las autoridades indígenas. Por otro lado, los(as) docentes oficiales señalaron al CRIC de privatizar la educación, al sentir en peligro sus espacios y derechos laborales, con la contratación de profesores(as) por otra entidad que no fuera el Estado (entrevista a José Campo, 2012). Entonces la normatividad educativa y el proceso de contratación docente influyeron en el distanciamiento entre los(as) docentes y las organizaciones y cabildos indígenas.

No obstante la contratación docente generó una dilatación en la relación que tenían los docentes y la comunidad, al haber ahora docentes contratados por la SED (provisionales), por el Ministerio de Educación (propiedad) y los(as) docentes contratados por la organización indígena regional (oferentes), estableciendo una diversificación docente (entrevista a José Campo, 2012). Si bien estos últimos docentes serían contratados con otras condiciones laborales, el propósito era que los(as) profesores(as) oferentes empezaran a cuestionar los proyectos pedagógicos que se habían desarrollado en las instituciones educativas en donde iban a trabajar (entrevista a José Campo, 2012).

\section{EL PEC}

Aunque el PEC llegó a los cabildos indígenas de la zona oriente en la década de 1990 a través del PEBI, el COTAINDOC solo avanzaría en una propuesta de construcción del PEC hasta el 2004. Ese año surgieron algunas discusiones sobre cómo debería ser el quehacer del Programa de Educación sobre el PEC, planteando la idea de diseñar un proyecto educativo a nivel zonal o en conjunto para todas las escuelas de los territorios indígenas de la zona oriente. 
Sin embargo esta idea se cuestionaría debido a que no reconocía la situación y contexto del COTAINDOC, en donde se reunían cinco pueblo indígenas. Frente a esta propuesta se planteó que la construcción del PEC se hiciera desde cada pueblo indígena, a partir de la participación activa de cada comunidad (Programa de Educación, 2003, pp. 15-17). Construir el PEC implicaría entonces el reconocimiento y el trabajo de una comunidad sobre su afirmación étnica, sobre sus problemas y sus espacios comunitarios. Con todo estos durante los primeros años, el desarrollo que tuvo el PEC en el programa correspondió, no tanto al fortalecimiento del cabildo y de las autoridades indígenas (aunque se le relacionará con estas) sino, al posicionamiento de las propuestas educativas de la organización indígena regional.

\section{La proyección del COTAINDOC y la trasformación del Programa de Educación}

Tanto el quehacer del Programa de Educación, como estas propuestas, se trasformaron debido a las nuevas dinámicas y a la nueva posición que vivió el COTAINDOC desde el 2006 hasta el 2011. En primera medida, el programa de educación creció intentando vincular a diferentes lideres o representantes de cada comunidad indígena ${ }^{11}$. A esto debemos agregarle que las comunidades y organizaciones indigenas estaban viviendo un momento de vicisitud en torno a la educación, por tanto que la diversificación docente ${ }^{12}$, la necesidad de (re)construir el PEC y los convenios inter-administrativos fueron aspectos que generaron que los cabildos le solicitaran al Programa de Educación, orientación política y administrativa en los procesos educativos (entrevista a Jorge Sánchez, 2012). Estas tensiones y situaciones se condensaron con la insistente intención del consejero mayor del COTAINDOC, Jorge Sánchez, por fijar el lugar y la acción de la organización zonal sobre el tema educativo. Así el propósito del Programa de Educación,

${ }_{11}$ En el 2006 se nombró a Silvia Jimena Muelas como coordinadora política del programa de educación, a Heliodoro Quijano como coordinador pedagógico y a Dilio Pillimué y al antropólogo Mauricio Prada como apoyos pedagógicos profesionales.

12 En la zona oriente había 389 maestros de los cuales 208 eran de propiedad, 173 provisionales y 38 oferentes (COTAINDOC, 2011), 
durante el 2006 y el 2011, fue asentar una decisión y un lugar por parte de las autoridades indígenas sobre la situación de la educación en los territorios indígenas (entrevista a Jorge Sánchez, 2012).

El programa cambió su quehacer desarrollando diferentes encuentros con cada una de las comunidades indígenas en donde se socializaron y discutieron algunos aspectos de la propuesta de educación propia. Los encuentros se hicieron contrastando las politicas educativas de las instituciones estatales con lo que significaba ser indígena y el aprendizaje cultural (entrevista a Silvia Muelas, 2012; Programa de Educación, 2006b). Así las cosas, el programa se encontró con dos situaciones educativas diferentes. Por un lado estaban los territorios indígenas de Quichaya, La Gaitana, Tumburáo y La María que manejaban solo una estructura organizativa/administrativa centralizada en un proyecto pedagógico comunitario (PEC); y por otro lado, estaban los resguardos de Polindara, Ambaló, Kizgó, Pitayó, Paniquitá en donde cada territorio tenía de dos a cuatro instituciones educativas independientes desarrollando diferentes proyectos educativos que partían de la relación con el director de núcleo y de la SED (Entrevista a Silvia Muelas, 2012).

Lo anterior llevó al programa a comprender que la estructura organizativa educativa ${ }^{13}$ intervenía en la relación que había entre la institución educativa y la comunidad; como también en la relación docente-comunidad. Entonces el Programa de Educación concluyó que su quehacer, más que unos talleres, unas estadísticas o un posicionamiento, debía ser una formación politica constante con las comunidades y organizaciones indígenas locales (Entrevista a Silvia Muelas, 2012).

\section{Docentes y maestros(as) como dinamizadores(as) comunitarios(as)}

A partir de estas situaciones se replanteó la relación entre las organizaciones indígenas y los docentes. El Programa de Educación ya no

\footnotetext{
13 La estructura organizativa de la educación supone el número de proyectos e instituciones educativas los espacios educativos y la comunidad educativa tradicional (docentes, alumnos y rectores).
} 
volvió a ver a los(as) docentes como sujetos antagónicos a la afirmación de la identidad étnica, sino como dinamizadores de los procesos comunitarios y como figuras trasformadoras de la situación educativa, siendo ahora actores válidos en la construcción del PEC (Entrevista a Carlos Sánchez, 2012). Sin embargo los docentes siguieron manteniendo relaciones de tensión con las organizaciones indígenas, pues al ser considerados sujetos para la trasformación de la situación educativa encarnaban las condiciones ambivalentes que se dan cuando se cohesionan las propuestas de las organizaciones indígenas y el sistema educativo nacional. Por ello, por el momento solo los docentes oferentes fueron quienes se apropiaron de las nuevas dinámicas con las comunidades indígenas que proponía el Programa de Educación.

\section{Diplomados y evaluaciones}

Se hicieron dos diplomados sobre la construcción del PEC con los docentes de la zona oriente. El primer diplomado fue "Los Proyectos Educativos Comunitarios en el marco de los Planes de Vida" el cual se hizo con los docentes contratados por el CRIC y comprendió de tres encuentros presenciales. El primer encuentro consistió en una contextualización sobre los pueblos indígenas de la zona oriente del Cauca, donde se les preguntó a los(as) docentes qué entendían por el CRIC, el COTAINDOC y el Cabildo, cuáles eran los principios y reivindicaciones de las organizaciones indígenas y cuáles eran los pueblos indígenas de la zona oriente.

En el segundo y tercer taller se tocó el tema de ¿qué es el PEC? y ¿Cómo se construye?, comparando los conceptos, fundamentos y finalidades entre el Proyecto Educativo Institucional y el PEC (Programa de Educación, 2007a). Esto le permitió al programa entender que varios docentes no sabian cuántos resguardos y pueblos indigenas habían en la zona oriente, no concebían la importancia de los planes de vida y el PEC ni conocían las organizaciones indígenas de la región (entrevista a Silvia Muelas, 2012).

Un años después, en el 2008 se desarrolló el diplomado "Currículo propio en el marco del PEC" con la intención de conocer la experiencia de los docentes de la zona oriente y de otros lugares sobre la construcción 
del currículo. Alli se trabajaron con los(as) profesores(as) oferentes, los aspectos pedagógicos y políticos alcanzados por ellos en su quehacer, como también el lugar del currículo alcanzado en la construcción del proyecto educativo comunitario (Programa de Educación, 2008b).

A partir de las reflexiones ocasionadas en estos espacios, el Programa de Educación estableció la necesidad de hacer un ejercicio de control territorial y comunicativo sobre los(as) docentes, para lo cual se planteó que las autoridades indígenas definieran los principios y procedimientos para la selección, traslado y desvinculación docente (Programa de Educación, 2008a; COTAINDOC, 2008) ${ }^{14}$. Estos criterios y procedimientos representaron, como primera medida, la trasformación de la relación entre docentes (oferentes) y las autoridades indigenas.

\section{Memorias Pedagógicas o Bitácoras}

Las bitácoras surgieron por iniciativa del Programa de Educación, entre 2006 y 2007, con la intención de conocer las realidades y situaciones de las instituciones educativas desde la interpretación de los(as) docentes, a través de la narración por parte del docente de las actividades pedagógicas que cotidianamente realizaba en su espacio laboral (Programa de Educación, 2007b). Esta propuesta fue apropiada por los(as) docentes oferentes, tanto que sugirieron cambiarle el nombre al programa por el de Memorias Pedagógicas.

La Memoria Pedagógica le permitió al programa entender algunos problemas del modelo educativo actual, como la manera en que lo(as) docentes manejaban la temática educativo, el currículo académico, los proyectos pedagógicos y la relación de estos con los(as) niños(as), con las comunidades y con las autoridades indígenas. Por ejemplo, Silvia Muelas recuerda que algunos maestros, aunque colocaban en las bitácoras todo lo que hacian en la clase que dictaban, nunca relacionaban

\footnotetext{
${ }^{14}$ Algunos criterios para la selección docente fueron: haber presentado servicio al cabildo, tener conocimientos académicos y del proceso organizativo indígena; el o la docente debe ser capaz de asumir un diálogo integral con los diferentes componentes del plan de vida de los pueblos indigenas, conocer de legislación y cosmovisión indígena (COTAINDOC, 2008). Y los procedimientos para su selección y traslado son: el cabildo postula y controla el quehacer del docente, el docente debe ser reconocido, avalado y evaluado por la comunidad indígena del territorio donde trabaje (COTAINDOC, 2008).
} 
su quehacer con la comunidad, con la autoridad o con las organizaciones indigenas (Entrevista a Silvia Muelas, 2012).

En 2008 con el cambio de coordinación del programa, cambió la intención y la forma de las Memorias Pedagógicas. Al principio no había condiciones o elementos temáticos centrales para su elaboración, pero desde ese año las Memorias Pedagógicas se reestructuraron enfocándose en un trabajo más específico sobre los procesos y prácticas educativas de las escuelas (Entrevista a Silvia Muelas, 2012) ${ }^{15}$. Justamente los criterios de las Memorias Pedagógicas se concentraron en dos aspectos: por un lado se dio una reflexión sobre la relación entre los conocimientos y las prácticas educativas que desarrollaba el docente y los espacios y propuestas de las autoridades indígenas; y por otra parte, una reflexión pedagógica sobre la manera en que el quehacer del docente se sincretizaba con las situaciones que vivian los niños, las niñas, los padres de familia y la comunidad (Programa de Educación, 2008a).

\section{El PEC y la educación desde la diversidad}

En el 2007 las autoridades indígenas determinaron que la construcción del PEC se debía hacer por territorio y comunidad indigena, por lo tanto se planteó que cada proyecto educativo se elaboraría a partir de su relación con el gobierno y la autoridad indígena (asambleas y cabildos), la identidad étnica ( $\mathrm{El}$ ser nasa, misak, ambalueño, etc.) y la unidad de los pueblos indígenas (Programa de Educación, 2006a), aspectos que no estaban articulados en la educación oficial.

No obstante, en los diferentes encuentros que el programa desarrolló para reflexionar sobre la construcción del PEC surgió la discusión sobre si este (PEC) debía sujetar solo los aspectos o contextos culturales de los pueblos indígenas o si debía dialogar con los conocimientos tradicionalmente institucionales de la educación oficial.

\footnotetext{
15 Se determinaron como criterios para la construcción de las bitácoras: "la concepción y los avances PEC; la articulación y la relaciones con la comunidad; las prácticas pedagógicas; los compromisos $\mathrm{y}$ aporte al proceso organizativo; las actitudes y experiencia investigativa; las proyecciones y autocrítica; escritura, redacción y presentación” (Programa de Educación, 2007b).
} 
Frente a esto, el mayor Dillio Pillimuet afirmaba que la educación propia no dejó de interactuar con el conocimiento occidental que se desarrollaba en la escuela oficial, al contrario:

[...] hay que confrontar esos dos conocimientos de acuerdo a la realidad, al contexto en que nos movemos y cómo los contextos son permanentemente cambiantes. Entonces por eso en el PEC no podíamos dejar de hablar de cosas concluidas, de cosas absolutas, sino que son permanentemente flexibles y que permanentemente están en construcción. (Entrevista a Dillio Pillimuet, 2012)

Entonces un aspecto interesante que el trabajo sobre el PEC implicó, fue la reflexión por parte de las organizaciones y autoridades indígenas sobre cuáles eran los espacios y los contenidos que la educación indígena debería sujetar. Se cuestionó el lugar central que tenían el aula, el salón y el maestro en los procesos pedagógicos y de trasmisión de conocimiento. Y se ahondó en la búsqueda de otros espacios para la enseñanza y el aprendizaje, como los espacios y actividades comunitarias y organizativas (Entrevista Joaquín Tombé, 2012). Sin embargo el Programa de Educación avanzó con lentitud en la construcción de los PEC, desarrollando hasta entonces (2010) una guía de sistematización de los estados de los PEC con aspectos como el diagnostico, la justificación, la misión, la visión, etc. (COTAINDOC, 2007a).

\section{Consideraciones}

Al mirar el surgimiento y el desarrollo del Programa de Educación del COTAINDOC, a través de dos de sus principales propuestas (el trabajo con docentes y el PEC), es posible encontrar que la diversidad cultural y/o étnica asume un papel importante en la reconstrucción de la relación entre los procesos educativos y las autoridades indígenas; lo cual repercutió en las propuestas educativas y en las relaciones organizativas que tienen el CRIC, el COTAINDOC y los cabildos y comunidades. 
Al momento de surgir el COTAINDOC, el Programa de Educación desarrolló su quehacer con el propósito de posicionar esta organización. Tanto sus actividades como el significado de la educación se fundamentaron en identificar una historia, unos elementos y unos espacios que permitieran enlazar a doce comunidades indígenas en una organización zonal. Sin embargo, el Programa de Educación se involucró más en los espacios que estaba desplegando el CRIC con el Estado y menos en la generación de procesos de reconocimiento, reflexión y discusión entre las comunidades indígenas. Por ejemplo, se afirmaba que para potenciar la educación propia era necesario partir del acompañamiento de la organización regional, posicionando la historia y las propuestas del CRIC, las cuales eran cuestionadas o desconocidas por la SED, los rectores y algunas comunidades indígenas (Entrevista Eliodoro Quijano, 2012).

No obstante, el Programa de Educación entendió que los procesos educativos respondian a las dinámicas de las autoridades y comunidades indígenas a nivel local, lo que implicó que el quehacer del programa se re-adecuara a las dinámicas de cada cabildo o comunidad. Por ejemplo, el programa identificó que varios proyectos educativos se habían diseñado solamente como requerimiento para la creación de escuelas ante el Estado, lo que confirmaba su idea sobre la disyuntiva ente los espacios educativos institucionales y las dinámicas sociales y organizativas de los pueblos indígenas (Programa de Educación, 2003). Aquí, se puede ver la intención del Programa de Educación del COTAINDOC por empezar un proceso de redefinición de sus propias dinámicas y espacios organizativos, como también de sus reivindicaciones.

Lo anterior es importante debido a que manifiesta que las organizaciones indígenas no son sujetos homogéneos y pasivos. De hecho lo primero a entender es que el Programa de Educación del COTAINDOC trasformó su quehacer, su propósito y su relación frente a otras organizaciones y entidades; lo que pondría en cuestión los proyectos y los propósitos de las mismas organizaciones indígenas. Esto quiere decir que aunque haya relaciones de padrinaje y acompañamiento entre las mismas organizaciones indígenas, no se puede pasar por alto la configuración de múltiples relaciones, discusiones e intereses entre 
estas, como se puede ver en el caso del Programa de Educación del COTAINDOC.

En este caso la intención de líderes, lideresas y comunidades indígenas por gestar -en cierta media- la construcción de una conciencia étnica colectiva o, en otras palabras, un nuevo espacio y sujeto colectivo interétnico, el COTAINDOC, modificó el propósito y el quehacer del Programa de Educación. Este se reconfiguró para forjarse en un espacio que potencializa el encuentro entre los diferentes pueblos y organizaciones indígenas de la zona oriente, con la intención de posicionar el reconocimiento de la diversidad étnica de la zona y trasformar las relaciones y los diálogos entre las instituciones educativas, las comunidades indígenas de la zona oriente y la organización indígena regional.

Lo anterior implicó desarrollar espacios que permitieran la reflexión colectiva sobre la situación educativa y la discusión de algunas propuestas del CRIC. Para esto el Programa de Educación trasformó su relación con algunas organizaciones indígenas y estatales, como también su dinámica con los cabildos, las comunidades indígenas y los docentes. Esto lo podemos ver a través de dos procesos: por un lado, en la relación entre las autoridades indígenas y los procesos educativos; y segundo, a partir del lugar que se le dio al reconocimiento étnico y a la diversidad cultural.

Por un lado, el programa trasformó su quehacer en un espacio de formación política cotidiana, el cual consistió en crear espacios y procesos de constante diálogo con las autoridades indígenas sobre los problemas específicos de las comunidades y de las situaciones educativas. La formación politica implicó alterar las relaciones culturales y políticas que históricamente los pueblos indigenas habian tenido con el ámbito escolar y educativo. Por ejemplo, se vincularon los procesos administrativos y pedagógicos de las instituciones educativas con los espacios de participación de las comunidades indígenas (Entrevista a Dillio Pillimuet, 2012). Para esto se propuso que las autoridades indigenas aplicaran gobernabilidad y autonomía en los procesos educativos de los territorios indigenas a partir de la construcción del PEC; de la selección, traslado y evaluación docentes; y 
mediante el diseño de los currículos y planes de inversión de los establecimientos educativos (COTAINDOC, 2007b).

Asimismo la formación politica implicó que los(as) profesores(as) oferentes empezaran a participar activamente en espacios de diálogo y formación con las autoridades y organizaciones indígenas (como en las Memorias Pedagógicas y Diplomados). El encuentro con los docentes representó la trasformación de la manera en que estos eran identificados desde las organizaciones indígenas -como actores antinómicos del reconocimiento étnico-, para luego identificarlos como elementos importantes en la participación comunitaria y en la autonomía educativa ejercida por las autoridades indígenas. Se dio reconocimiento así de una diversificación en el quehacer y control docente.

Otro aspecto importante del trabajo del Programa de Educación fue el reconocimiento de la diversidad de los pueblos indígenas en la zona oriente. Lo que sucedía era que no todas las comunidades indígenas de la zona oriente habian sido reconocidas por el Estado y por las mismas organizaciones indígenas, pues las comunidades de Polindara, Kizgò y Ambaló estaban hasta ahora (re)definiendo, (re)identificando y legitimando las características que tenían como pueblos indígenas; mientras que otras ya lo habían hecho (misak y nasa). Si el COTAINDOC tenía la necesidad de posicionarse y enlazar a las comunidades indigenas con el CRIC, no lo iba a hacer desconociendo la realidad cultural y social de la zona oriente. Al contrario, la diversidad étnica sería un elemento trascendental en el quehacer del Programa de Educación y en el posicionamiento del COTAINDOC.

El Programa de Educación se reordenó con la intención de una participación más activa de los diferentes pueblos indígenas, a través de sus representantes o delegados. Esto no fue percibido como un problema sino como una fortaleza, ya que la diversidad étnica enriquece los espacios propuestos por las comunidades y autoridades indigenas alrededor del proceso educativo (Entrevista a Jorge Sánchez, 2012). Un ejemplo de lo anterior fue el PEC. Este no se hizo de manera zonal -como en otras organizaciones indígenas del Cauca- sino a partir del reconocimiento y el trabajo con los diferentes pueblos indígenas dentro del COTAINDOC. Así el acompañamiento en la construcción 
del PEC se dio desde cada grupo étnico -cada comunidad y cabildo indígena- con la intención de fortalecer los procesos organizativos y de reconocimiento étnico de los pueblos indígenas de la zona oriente.

En general la diversidad le permitió al Programa de Educación trasformar su quehacer; las dinámicas que establecía con los docentes y con las comunidades y cabildos indigenas y generar espacios de reflexión sobre las propuestas que venían de la organización indígena regional. Es decir, para que las reivindicaciones y propuestas de la educación propia tuvieran eco en la zona oriente, el Programa de Educación vio necesario formar una conciencia étnica colectiva que permitiera a las comunidades y autoridades indígenas reconocerse y re-construirse como pueblos indigenas en medio de la apropiación del modelo educativo oficial y de las nuevas situaciones que se habian generado tras las negociaciones CRIC-Estado.

Lo expuesto va más allá de las instituciones educativas y pone en discusión la relevancia y la articulación que tienen los tipos de proyectos educativos que se proponen desde el Estado y desde las mismas organizaciones indígenas, poniendo en cuestión la manera en que se estaba llevando a cabo el reconocimiento étnico y/o de la diferencia dentro de las organizaciones indígenas y espacios educativos.

Lo que hizo el Programa de Educación fue implementar los procesos y propuestas educativas antes descritas articulándolas con las situaciones que vive la población en donde se iban a desarrollar, sometiendo a reflexión la pertinencia y la articulación de las propuestas educativas del Estado y del CRIC con la realidad que vivían las comunidades indígenas de la zona oriente.

Lo anterior no quiere decir que las propuestas del Programa de Educación del COTAINDOC sean perfectas y no tengan tensiones, pues -como se expuso- durante los primeros diez años del COTAINDOC las propuestas y las dinámicas del Programa de Educación estuvieron envueltas en conflictos y cambios; y mucho menos se puede aseverar que sus espacios y reivindicaciones no tengan una relación con la lógica de gobierno indirecto que desplegó el Estado. Por el contrario, se manifiesta que el Programa de 
Educación, a la luz de contextos locales y diversos, pretenden constituirse como un escenario en donde se pone en cuestión la forma como se reconoce lo étnico y/o la diferencia en las políticas etno educativas del Estado, como también al momento de apropiar o aplicar las propuestas educativas del CRIC.

Teniendo en cuenta que lo revisado anteriormente no manifiesta que todas las personas que participan en el COTAINDOC piensen de la misma manera, se considera que el Consejo Territorial de Autoridades Indígenas del Oriente Caucano y el Programa de Educación debe reflexionar sobre su trasegar y su relación, tanto con las instituciones estatales como con las mismas organizaciones indígenas, ya que estas organizaciones zonales -que tienen una mayor relación con las comunidades y realidades locales- deben desarrollar su quehacer a partir de este tipo de reflexión en el cual la diversidad es una especie de filtro frente a propuestas e ideas que no se articulan con la realidad, las dinámicas y prácticas locales. Sin eso significar una división organizativa.

\section{Referencias}

Barfield, T. (2001/1997). Diccionario de Antropología. España: Ediciones Bellaterra.

Barth, F. (1976/1969). Introducción. En: Grupos étnicos y sus fronteras (pp. 9-49). México: Fondo de Cultura Económico.

Bartolomé, M. (2004/1997). Gente de costumbre y gente de razón: Las identidades étnicas en México. México: Siglo Veintiuno Editores.

Bartolomé, M. (2006). Interculturales. Antropología Politica del Pluralismo cultural en América Latina. México. Siglo Veintiuno Editores.

Bonfil Batalla, G. (1987a). Una tierra con civilización milenaria. En México Profundo: Una civilización negada (pp. 22-43). México: Grijalbo. 
Bonfil Batalla, G. (1987b). Lo indio desindianizado. En México Profundo: Una civilización negada (pp. 73-96). México: Grijalbo.

Caviedes, M. (2002, enero-diciembre). Solidarios frente a colaboradores. Antropología y movimiento indígena en el Cauca en la décadas de 1970 y 1980. Revista Colombiana de Antropología, 38, 237-260.

COTAINDOC (2000a, diciembre). Acta General del Primer Congreso del COTAINDOC, La María. Acta interna del COTAINDOC. Silvia, Cauca.

COTAINDOC (2000b, junio). Acta de la Junta Directiva Ampliada de la Zona Oriente, Paniquita 7 y 8 de Junio del 2000. Acta interna del COTAINDOC. Silvia, Cauca.

COTAINDOC (2002). Análisis General del Comité de Educación. Documento interno del COTAINDOC. Silvia, Cauca: Inédito.

COTAINDOC (2006, mayo). Memoria del IV Congreso del Consejo Territorial de Autoridades Indígenas del Oriente Caucano, del 14 al 17 de Mayo del 2006. Documento interno del COTAINDOC. Silvia, Cauca.

COTAINDOC (2007a, junio). Memoria de la Junta Directiva de Educación de Polindara en el 2007. Documento interno del COTAINDOC. Silvia Cauca.

COTAINDOC (2007b, junio). Mandato de Polindara sobre la Autonomía Educativa. Documento oficial del COTAINDOC. Silvia, Cauca.

COTAINDOC (2008, julio). Memorias del V Congreso del COTAINDOC. Documento interno del COTAINDOC. Silvia, Cauca.

COTAINDOC (2011). Conclusiones Generales de los Congresos Zonales para el trabajo de la Junta Directiva de Paniquita. Silvia, Cauca.

CRIC, Consejo Regional Indígena del Cauca (2004). ¿Qué Pasaría si la Escuela...? 30 años de construcción de una propuesta de educación propia. Popayán: Programa de Educación Bilingüe e Intercultural. 
CRIC, Consejo Regional Indígena del Cauca. (2006). Mecanismo de Articulación Local, Zonal y Regional. Documento Interno.Popayán.

CRIC, Consejo Regional Indigena del Cauca. (2011). Sistema Educativo Indigena Propio. Primer Documento de Trabajo. Programa de Educación Bilingüe e Intercultural. Popayán: CRIC.

Díaz-Polanco, H. (2009). Para entender la diversidad cultural y la autonomía en México. China: Nostra Ediciones.

Findji, M. T. (1993). Tras las huellas de los paeces. En Vasco, L.G. (Ed.). Encrucijadas de Colombia Amerindia (pp. 49-70). Bogotá: ICANH.

Friede, J. (1972). El indio lucha por la tierra. Colombia: Ediciones la Chispa.

Gros, C. (1991). Colombia Indigena. Identidad cultural y cambio social. Bogotá: CEREC.

Gros, C. (1997). Identidad, Estado y Modernidad. En Restrepo, E. y Uribe, M. V. (Eds.). Antropología en la modernidad. Colombia: ICANH.

Gros, C. (1999, enero-abril). Ser diferente por (para) ser moderno. Análisis Político (36), 3-20.

Gros, C. y Morales, T. (2009). ¡A mí no me manda nadie! Historia de vida de Trino Morales. Bogotá: ICAHN.

Hall, S. (2003). ¿Quién necesita la identidad? En Hall y Du Gay (Eds.). Cuestiones de Identidad cultural (pp. 13-39). Buenos Aires: Amarrortu Editores.

Hougton, J. y Villa, W. (2005). Violencia Politica contra los pueblos indigenas en Colombia 1974-2004. Bogotá: OIA-CECOCIN.

Laurent, V. (2005). Surgimiento y auge del movimiento indígena en Colombia. En Comunidades indígenas, espacios políticos y movilización electoral en Colombia, 19990-1998. Motivaciones, campos de acción e impactos (pp. 67-114). Bogotá: IFEA. ICANH. 
Neira, C. (2008). ¿Qué son los movimientos étnicos?. En Gutiérrez, D. y Clausen H. (Coords.). Revisitando la etnicidad: miradas cruzadas en torno a la diversidad (pp. 71-81). México: Siglo Veintuno Editores.

Pancho, A. (2007). Una decisión comunitaria para avanzar en la autonomía. Çxatu'çe Revista de Etnoeducación, (13), 12-16.

Programa de Educación (2003). Lineamientos Políticos y de Trabajo del Programa de Educación, Área Socio Cultural. Silvia, Cauca: COTAINDOC, inédito.

Programa de Educación (2006a). Lineamientos Políticos y de Trabajo del Programa de Educación. Silvia, Cauca: COTAINDOC, inédito.

Programa de Educación (2006b). Informe del Equipo Zonal del COTAINDOC al PEBI sobre los objetivos planteados para el 2006. Silvia Cauca: COTAINDOC, inédito.

Programa de Educación (2007a). Programa del Diplomado: Los proyectos educativos comunitarios en el marco de los planes de vida. Silvia, Cauca: COTAINDOC, CRIC y UAIIN, inédito.

Programa de Educación (2007b). Guía de trabajo de las Bitácoras para la zona oriente. Silvia, Cauca: COTAINDOC, inédito.

Programa de Educación (2008a). Análisis de la Situación: La Política Educativa y el Proyecto de Autonomía. Silvia, Cauca: COTAINDOC, inédito.

Programa de Educación (2008b). Taller de análisis y evaluación de productos presentados por los docentes oferentes. Silvia, Cauca: Diplomado de Currículo propio en el marco del PEC. COTAINDOC, PEBI y UAIIN, inédito.

Programa de Educación (2010). Documento interno elaborado por parte del equipo de educación en el 2010 para la página web del COTAINDOC. Silvia, Cauca: Inédito.

Rappaport, J. (2008). Utopías interculturales. intelectuales públicos, experimentos con la cultura y pluralismo étnico en Colombia. Bogotá:. Editorial Universidad del Rosario. 
Rueda, R. A., Gómez, C. y Martínez, M. A. (2003). Proyectos Educativos Comunitarios en Pueblos Indigenas. Colombia: FUCAI. CORDAID.

Vasco, L. G. (2002). Acompañando la organización y la lucha indígena. En Entre selva y paramo, viviendo y pensando la lucha india. Bogotá: ICANH. Disponible en: http:/ / www.luguiva.net/libros/detalle.aspx?id=3.

\section{Cómo citar este artículo}

Gutiérrez-Sánchez, S. A. (2015). La diversidad en las organizaciones indígenas del Cauca: El Consejo Territorial de Autoridades Indigenas del Oriente Caucano. Universitas Humanistica, 80, 293-322. http:/ /dx.doi.org/ 10.11144/Javeriana. UH80.doic 